\title{
Implementation of Image Compression using fast Wavelet Transform using HAAR and Daubechies Wavelets
}

\author{
Er. Neha Goyal ${ }^{1}$, Er. Monika Aggarwal ${ }^{2}$ \\ ${ }^{1,2}$ (Electronics \& Communication Engineering Department, B.G.I.E.T,Sangrur,India)
}

\begin{abstract}
Image compression is the application of Data compression on digital images. The objective of image compression is to reduce redundancy of the image data in order to be able to store or transmit data in an efficient form. Fast Wavelet Transform (FWT) highlights the benefit of a faster compression and faster processing as compared to DWT with higher compression ratios at the same time and reasonably good image quality. Fast Wavelet Transform has been used to perform image compression. Haar and Daubechies wavelets have been implemented and comparitive results of fast wavelet transform using both the wavelets have been performed and compared to the Discrete Wavelet Transform technique. Mallet Algorithm based fast wavelet analysis makes the use of extension of a given finite-length signal and removes the border effects due to convolution. Image quality is measured objectively, using peak signal-to-noise ratio or picture quality scale, and subjectively, using perceived image quality. These results provide a good reference for application developers to choose a good wavelet compression system for their application.
\end{abstract}

Keywords: Fast Wavelet Transform, Image Compression, Daubechies Wavelet, peak signal to noise ratio.

\section{Introduction}

Wavelet analysis represents the next logical step: a windowing technique with variable-sized regions. Waveletanalysis allows the use of long time intervals where we want more precise low-frequency information, and shorter regions where we want high-frequency information.
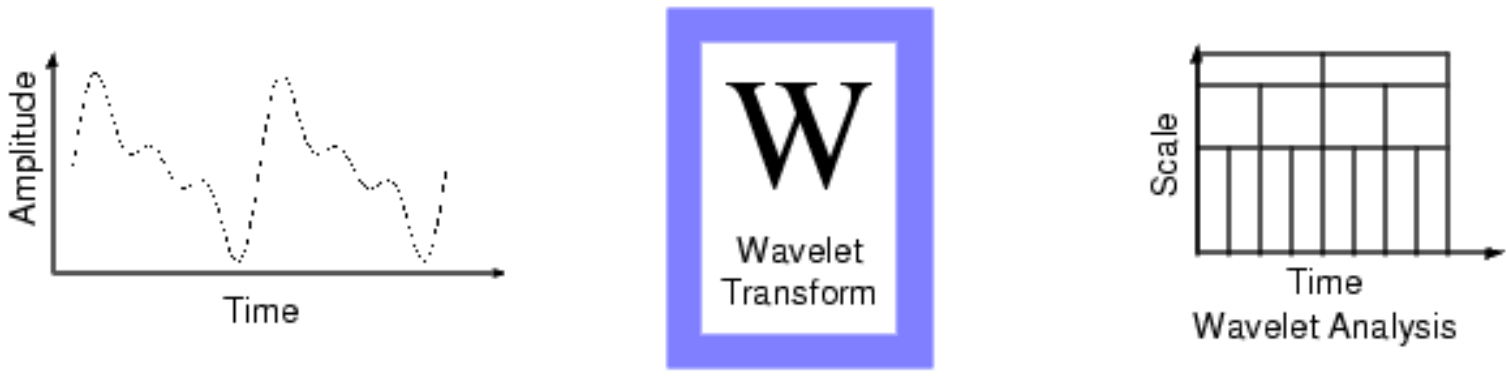

Figure 1: Wavelet Transform on a signal

Wavelet Transform in contrast with the time-based (eg. DFT), frequency-based (eg. FFT), and STFT (eg. DCT) views of a signal:

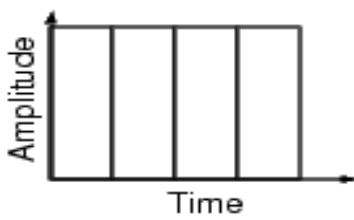

Time Domain (Shannon)

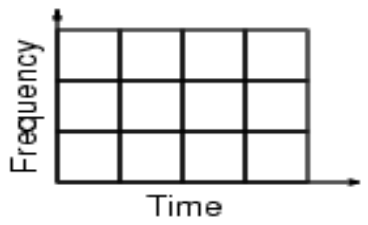

STFT (Gabor)

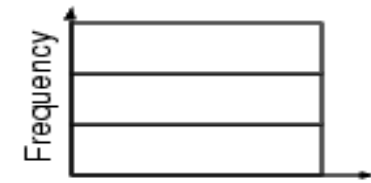

Amplitude

Frequen cy Domain (Fourier)



Wavelet Analysis

Figure 2: Comparision of Various Trasform Techniques 
The Fast Wavelet Transform is a mathematical algorithm designed to turn a waveform or signal in the time domain into a sequence of coefficients based on an orthogonal basis of small finite waves, or wavelets. The transform can be easily extended to multidimensional signals, such as images, where the time domain is replaced with the space domain. It has as theoretical foundation the device of a finitely generated, orthogonal multiresolution analysis (MRA). Transform coding is a widely used method of compressing image information. In a transform-based compression system two-dimensional (2-D) images are transformed from the spatial domain to the frequency domain. An effective transform will concentrate useful information into a few of the low-frequency transform coefficients.

The discrete wavelet transform (DWT) is a mathematical tool that has aroused great interest in the field of image processing due to its nice features. Some of these characteristics are:

1) It allows image multi resolution representation in a natural way because more wavelet subbands are used to progressively enlarge the low frequency subbands;

2) It supports wavelet coefficients analysis in both space and frequency domains, thus the interpretation of the coefficients is not constrained to its frequency behavior and we can perform better analysis for image vision and segmentation; and

3) For natural images, the DWT achieves high compactness of energy in the lower frequency subbands, which is extremely useful in applications such as image compression. The introduction of the DWT made it possible to improve some specific applications of image processing by replacing the existing tools with this new mathematical transform. The JPEG 2000 standard [1] proposes a wavelet transform stage since it offers better rate/distortion (R/D) performance than the traditional discrete cosine transform (DCT).

Unfortunately, despite the benefits that the wavelet transform entails, some other problems are introduced. Wavelet-based image processing systems are typically implemented by memory-intensive algorithms with higher execution time than other transforms. In the usual DWT implementation [2], the image decomposition is computed by means of a convolution filtering process and so its complexity rises as the filter length increases. Moreover, in the regular DWT computation, the image is transformed at every decomposition level first row by row and then column by column, and hence it must be kept entirely in memory. The lifting scheme [3,4] is probably the best-known algorithm to calculate the wavelet transform in a more efficient way. Since it uses less filter coefficients than the equivalent convolution filter, it provides a faster implementation of the DWT. Other fast wavelet transform algorithms have been proposed in order to reduce both memory requirements and complexity, like line-based [5] and block-based [6] wavelet transform approaches that performs wavelet transformation at image line or block level. These approaches increase flexibility when applying wavelet transform and significantly reduce the memory requirements. In this scheme, the 2D-DWT is performed in only one pass, avoiding multiple-layer transpose decomposition operations. One of the most interesting advantages of this method is that the computation of each wavelet subband is completely independent. An HVS is more sensitive to energy with low spatial frequency than with high spatial frequency. In numerical analysis and functional analysis, a discrete wavelet transform (DWT) is any wavelet transform for which the wavelets are discretely sampled. As with other wavelet transforms, a key advantage it has over Fourier transforms is temporal resolution: it captures both frequency and location information (location in time).

\section{Time Issues In Dwt}

Due to the rate-change operators in the filter bank, the discrete WT is not time-invariant but actually very sensitive to the alignment of the signal in time. To address the time-varying problem of wavelet transforms, Mallat and Zhong proposed a new algorithm for wavelet representation of a signal, which is invariant to time shifts. According to this algorithm, which is called a TI-DWT, only the scale parameter is sampled along the dyadic sequence $2^{\wedge} \mathrm{j}(\mathrm{j} \in \mathrm{Z})$ and the wavelet transform is calculated for each point in time.

\section{Discrete Wavelet Transform}

Wavelet transform (WT) represents an image as a sum of wavelet functions (wavelets) with different locations and scales [17]. Any decomposition of an image into wavelets involves a pair of waveforms: one to represent the high frequencies corresponding to the detailed parts of an image (wavelet function $\psi$ ) and one for the low frequencies or smooth parts of an image (scaling function $\varnothing$ ). DWT is a multi resolution decomposition scheme for input signals. The original signals are firs decomposed into two subspaces, low-frequency (low-pass) subband and high-frequency (high-pass) subband. For the classical DWT, the forward decomposition of a signal is implemented by a low-pass digital filter $\mathrm{H}$ and a high-pass digital filter $\mathrm{G}$. Both digital filters are derived using the scaling function $\Phi(t)$ and the corresponding wavelets $\Psi(t)$. The system downsamples the signal to half of the filtered results in the decomposition process. If the four-tap and non-recursive FIR filters with length L are considered, the transfer functions of $\mathrm{H}$ and $\mathrm{G}$ can be represented as follows

$\mathrm{H}(\mathrm{z})=\mathrm{h} 0+\mathrm{h} 1 \mathrm{z}-1+\mathrm{h} 2 \mathrm{z}-2+\mathrm{h} 3 \mathrm{z}-3$

$\mathrm{G}(\mathrm{z})=\mathrm{g} 0+\mathrm{g} 1 \mathrm{z}^{-1}+\mathrm{g} 2 \mathrm{z}-2+\mathrm{g} 3 \mathrm{z}-3$ 
The discrete wavelet transform (DWT) refers to wavelet transforms for which the wavelets are discretely sampled. A transform which localizes a function both in space and scaling and has some desirable properties compared to the Fourier transform. The transform is based on a wavelet matrix, which can be computed more quickly than the analogous Fourier matrix. Most notably, the discrete wavelet transform is used for signal coding, where the properties of the transform are exploited to represent a discrete signal in a more redundant form, often as a preconditioning for data compression. The discrete wavelet transform has a huge number of applications in Science, Engineering, Mathematics and Computer Science. Wavelet compression is a form of data compression well suited for image compression (sometimes also video compression and audio compression). The goal is to store image data in as little space as possible in a file. A certain loss of quality is accepted (lossy compression).Using a wavelet transform, the wavelet compression methods are better at representing transients, such as percussion sounds in audio, or high-frequency components in two-dimensional images, for example an image of stars on a night sky. This means that the transient elements of a data signal can be represented by a smaller amount of information than would be the case if some other transform, such as the more widespread discrete cosine transform, had been used. First a wavelet transform is applied. This produces as many coefficients as there are pixels in the image (i.e.: there is no compression yet since it is only a transform). These coefficients can then be compressed more easily because the information is statistically concentrated in just a few coefficients. This principle is called transform coding. After that, the coefficients are quantized and the quantized values are entropy encoded and/or run length encoded.

\section{Fast Wavelet Transform}

In 1988, Mallat produced a fast wavelet decomposition and reconstruction algorithm [Mal89]. The Mallat algorithm for discrete wavelet transform (DWT) is, in fact, a classical scheme in the signal processing community, known as a two-channel subband coder using conjugate quadrature filters or quadrature mirror filters (QMFs).

- The decomposition algorithm starts with signal $s$, next calculates the coordinates of $A 1$ and $D 1$, and then those of $A 2$ and $D 2$, and so on.

- The reconstruction algorithm called the inverse discrete wavelet transform (IDWT) starts from the coordinates of $A J$ and $D J$, next calculates the coordinates of $A J-1$, and then using the coordinates of $A J-1$ and $D J-1$ calculates those of $A J-2$, and so on. In order to understand the multiresolution analysis concept based on Mallat's algorithm it is very useful to represent the wavelet transform as a pyramid, as shown in figure 12. The basis of the pyramid is the original image, with $\mathrm{C}$ columns and $\mathrm{R}$ rows.

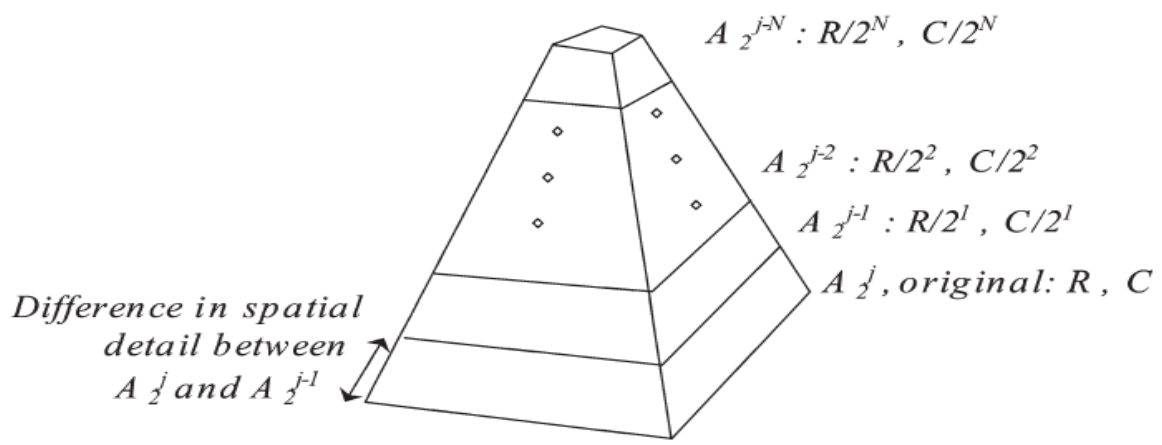

Figure 1: Pyramidal representation of Mallat's wavelet decomposition algorithm.

Given a signal $s$ of length $N$, the DWT consists of $\log 2 N$ stages at most. Starting from $s$, the first step produces two sets of coefficients: approximation coefficients $c A 1$, and detail coefficients $c D 1$. These vectors are obtained by convolving $s$ with the low-pass filter Lo_D for approximation, and with the high-pass filter Hi_D for detail, followed by dyadic decimation.

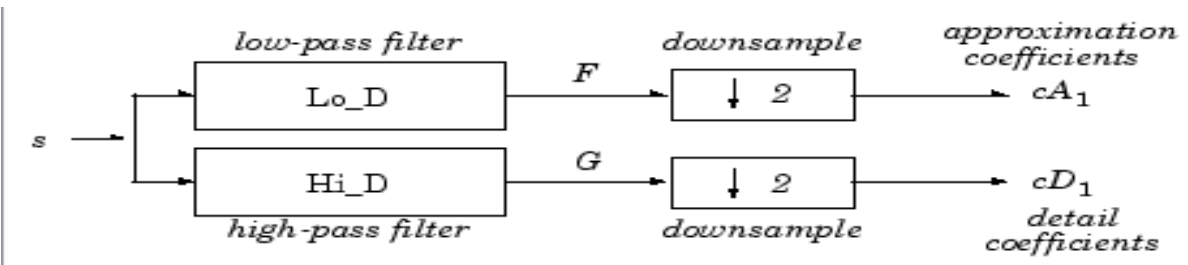

The length of each filter is equal to $2 n$. If $N=$ length $(s)$, the signals $F$ and $G$ are of length $N+2 n-1$, and then the coefficients $c A 1$ and $c D 1$ are of length 
floor $\left(\frac{(N-1)}{2}+n\right)$

The next step splits the approximation coefficients $c A 1$ in two parts using the same scheme, replacing $s$ by $c A 1$ and producing $c A 2$ and $c D 2$, and so on.

Classically, the DWT is defined for sequences with length of some power of 2, and different ways of extending samples of other sizes are needed. Methods for extending the signal include zero-padding, smooth padding, periodic extension, and boundary value replication. The basic algorithm for the DWT is not limited to dyadic length and is based on a simple scheme: convolution and down sampling [13]. As usual, when a convolution is performed on finite-length signals, border distortions arise. To remove these border effects, Fast Wavelet Transform was introduced. This algorithm is a method for the extension of a given finite-length signal [12].

\section{Multilevel Decomposition}

The decomposition process can be iterated, with successive approximations being decomposed in turn, so that one signal is broken down into many lower resolution components. This is called the wavelet decomposition tree.

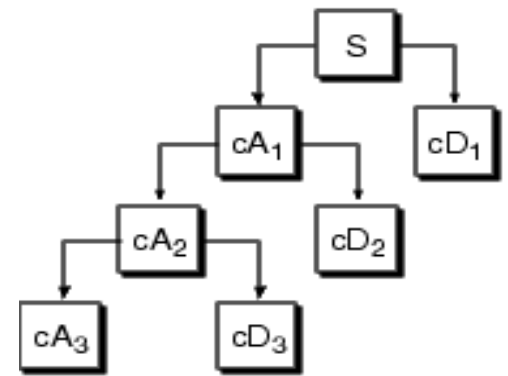

Figure 2: Multilevel Decomposition

Lifting schema of DWT has been recognized as a faster approach

- The basic principle is to factorize the polyphase matrix of a wavelet filter into a sequence of alternating upper and lower triangular matrices and a diagonal matrix.

- This leads to the wavelet implementation by means of banded-matrix multiplications

ALGORITHM follows a quantization approach that divides the input image in 4 filter coefficients as shown below, and then performs further quantization on the lower order filter or window of the previous step. This quantization depends upon the decomposition levels and maximum numbers of decompositionlevels to be entered are 3 for DWT



Figure 3: Wavelet Decomposition of Image

\subsection{WAVELET RECONSTRUCTION}

The filtering part of the reconstruction process also bears some discussion, because it is the choice of filters that is crucial in achieving perfect reconstruction of the original signal. The down sampling of the signal components performed during the decomposition phase introduces a distortion called aliasing. It turns out that 
by carefully choosing filters for the decomposition and reconstruction phases that are closely related (but not identical), we can "cancel out" the effects of aliasing

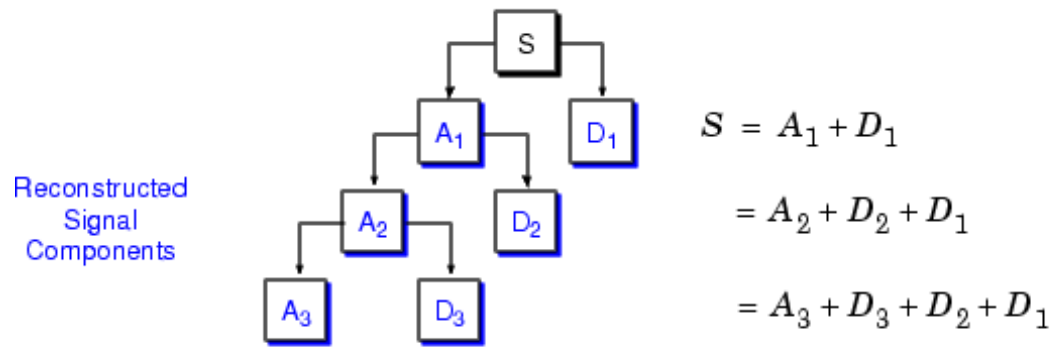

Figure 4: Wavelet Reconstruction

\section{Performance Parameters}

DWT and FWT based image compression has been performed to get the desired results of the proposed work. The work has been be done in MATLAB software using image processing and Wavelet toolbox that helps to exploit the various features of wavelet based image analysis and processing. Input image is to be compressed to a certain level using DWT / FWT based lifting and quantization scheme explained above by maintaining a good signal to noise ratio. Quantitative analysis have been presented by measuring the values of attained Peak Signal to Noise Ratio and Compression Ratio at different decomposition levels. The intermediate image decomposition windows from various low pass and high pass filters.

i. PSNR:

Peak Signal to Noise ratio used to be a measure of image quality. The PSNR between two images having 8 bits per pixel or sample in terms of decibels $(\mathrm{dBs})$ is given by:

PSNR $=10 \log 10$

mean square error (MSE)

ii. Compression Ratio:

Ratio of the size of compressed image to the input image is often called as compression ratio.

\section{Simulations And Results}

Image Compression is performed in the MATLAB software using wavelet toolbox. DWT and FWT based compression techniques have been implemented using lifting scheme and their results have been displayed in terms of qualitative analysis using image visual quality of input image, compressed image and reconstructed image and Quantitative analysis have been performed in terms of Elapsed time for both DWT and FWT and their respective compression ratios at third level of decomposition using Haar Wavelets

\section{QualitativeAnalysis}

\begin{tabular}{|c|c|c|c|c|}
\hline Wavelets used & Wavelet Type & $\begin{array}{c}\text { Compression Ratio } \\
(\%)\end{array}$ & $\begin{array}{c}\text { PSNR } \\
(\%)\end{array}$ & $\begin{array}{c}\text { Elapsed time for } \\
\text { processing(sec) }\end{array}$ \\
\hline \multirow{2}{*}{ Daubechies } & DWT & 47.4551 & 99.9822 & 0.703000 \\
\cline { 2 - 5 } & FWT & 47.4551 & 99.9822 & 0.562000 \\
\hline \multirow{2}{*}{ Haar } & DWT & 46.0973 & 99.9864 & 0.640000 \\
\cline { 2 - 5 } & FWT & 47.7269 & 99.9832 & 0.625000 \\
\hline
\end{tabular}

\section{QualitativeAnalysis}

1. Image Compression with Discrete Wavelet Transform at Level 3 Decomposition using Haar Wavelets
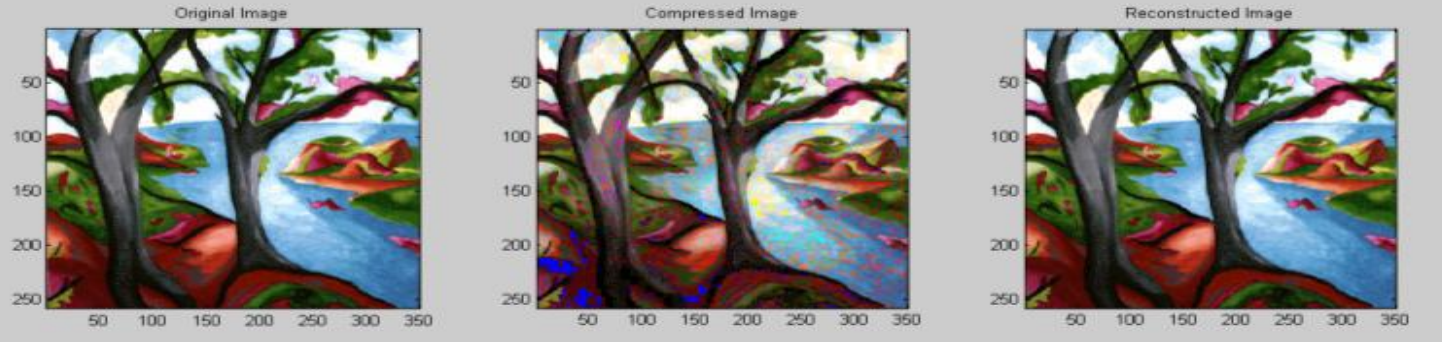

www.iosrjen.org 
2. Image Compression with Fast Wavelet Transform at Level 3 Decomposition using Haar Wavelets
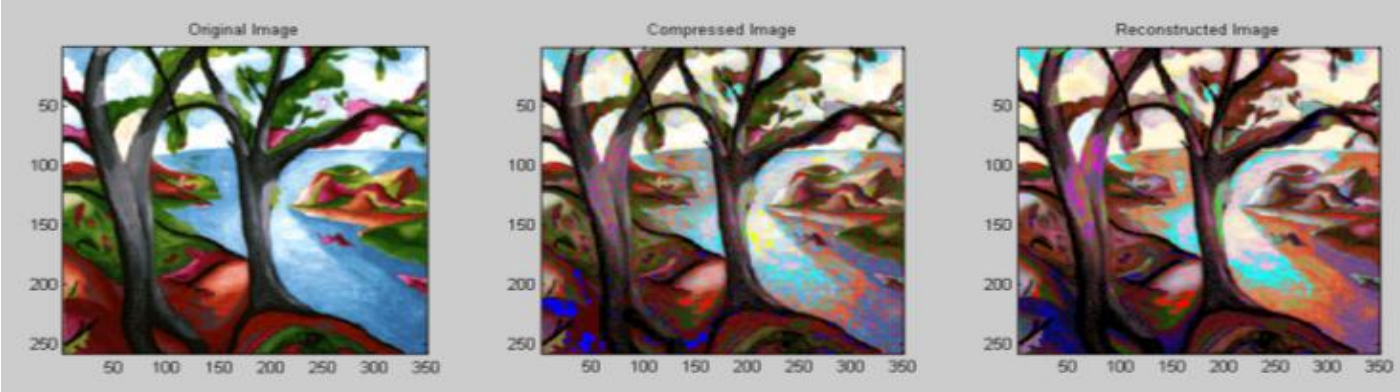

3. Image Compression with Discrete Wavelet Transform at Level 3 Decomposition using Daubechies Wavelets
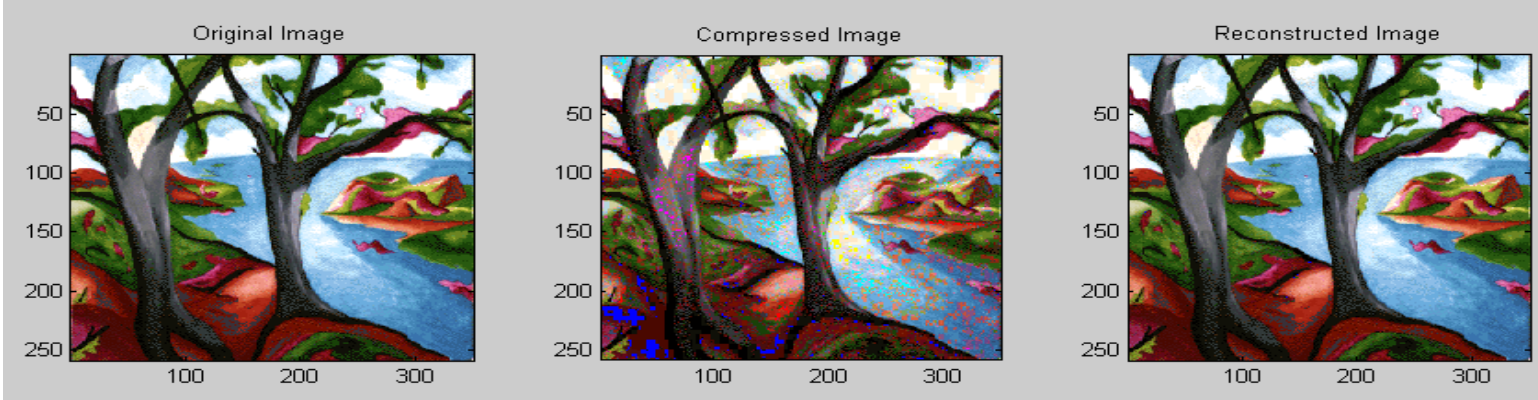

4. Image Compression with Fast Wavelet Transform at Level 3 Decomposition using Daubechies Wavelets
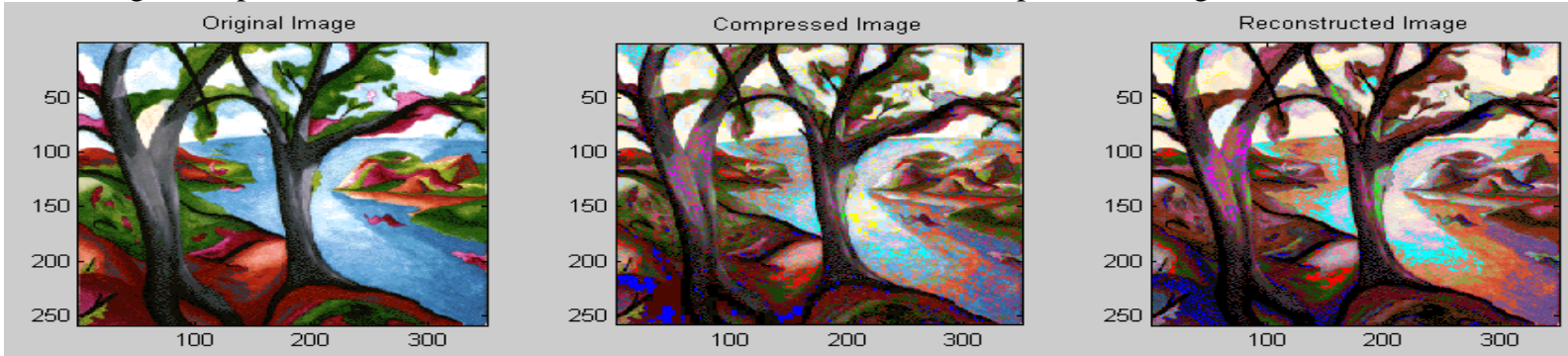

VIII. Conclusion And Future Scope

From the results, it has been concluded that image compression using fast wavelet transform shows results faster as compared to discrete wavelet transform with lesser processing time. Although picture visual quality or PSNR achieved with fast wavelet transform is slightly similar than that of discrete wavelet transform technique but the compression ratio achieved with fast wavelet transform is more than that of discrete wavelet transform technique. Comparing the types of wavelets used, Haar wavelet transform with Fast Wavelet transform shows the best results in terms of Compression Ratio and PSNR values. In future, other fast wavelet algorithms such as Atrous algorithm can be implemented or work may be done at implementation of this algorithm at chip level using VHDL or some other software.

\section{References}

[1] Image Compression using Wavelets: Sonja Grgc, Kresimir Kers, Mislav Grgc, University of Zagreb, IEEE publication, 1999

[2] M. Antonini, M. Barlaud, P. Mathieu, and I. Daubechies, Image coding using wavelet transform, IEEE Trans. Image Processing, vol. 1, pp.205-220, 1992.

[3] P.L. Dragotti, G. Poggi, and A.R.P. Ragozini, Compression of multispectral images by three-dimensional SPIHT algorithm, IEEE Trans. on Geoscience and remote sensing, vol. 38, No. 1, Jan 2000.

[4] Image compression using Fast Fourier Transform by Parminder Kaur, Thapar Institute of Engineering and Technology, Patiala.

[5] S-T. Hsiang and J.W. Woods, Embedded image coding using zeroblocks of subband/wavelet coefficients and context modeling, IEEE Int. Conf. on Circuits and Systems (ISCAS2000), vol. 3, pp.662-665, May 2000.

[6] Implementation of Data Compression and FFT on TinyOS: Ning Xu, Embedded Networks Laboratory, Computer Science Dept. USC. Los Angeles

[7] B. Kim and W.A. Pearlman, An embedded wavelet video coder using three-dimensional set partitioning in hierarchical tree, IEEE Data Compression Conference, pp.251-260, March 1997. 
[8] Y. Kim and W.A. Pearlman, Lossless volumetric medical image compression, Ph.D Dissertation, Department of Electrical, Computer,and Systems Engineering, Rensselaer Polytechnic Institute, Troy, 2001.

[9] J. Li and S. Lei, Rate-distortion optimized embedding, in Proc. Picture Coding Symp., Berlin, Germany, pp. 201-206, Sept. 10-12, 1997.

[10] E. Ordentlich, M. Weinberger, and G. Seroussi, A low-complexity modeling approach for embedded coding of wavelet coef_cients, in Proc. IEEE Data Compression Conf., Snowbird, UT, pp. 408-417, Mar. 1998.

[11] A.N. Netravali and B.G. Haskell, Digital pictures, representation and compression, in Image Processing, Proc. of Data Compression Conference, pp.252-260, 1997.

[12] S. Mallat, Multifrequency channel decompositions of images and wavelet models, IEEE Trans. Acoust., Speech, Signal Processing, vol. 37, pp.2091-2110, Dec. 1989.

[13] W.A. Pearlman, Performance bounds for subband codes, Chapter 1 in Subband Image Coding, J. W. Woods and Ed. Klvwer. Academic Publishers, 1991.

[14] Proposal of the arithmetic coder for JPEG2000, ISO/IEC/JTC1/SC29/WG1 N762, Mar. 1998.

[15] A. Said and W.A. Pearlman, A new, fast and ef_cient image codec based on set partitioning in hierarchical trees, IEEE Trans. on Circuits and Systems for Video Technology 6, pp. 243-250, June 1996.

[16] P. Schelkens, Multi-dimensional wavelet coding algorithms and implementations, Ph.D dissertation, Department of Electronics and Information Processing, Vrije Universiteit Brussel, Brussels, 2001.

[17] J.M. Shapiro, Embedded image coding using zerotrees of wavelet coef_cients, IEEE Trans. Signal Processing, vol. 41, pp.3445-3462, Dec. 1993.

[18] D. Taubman, High performance scalable image compression with EBCOT, IEEE Trans. on Image Processing, vol. 9, pp.1158-1170, July,2000.

[19] I.H. Witten, R.M. Neal, and J.G. Cleary, Arithmetic coding for data compression, Commun. ACM, vol. 30, pp. 520540, June 1987.

[20] J.W. Woods and T. Naveen, Alter based bit allocation scheme for subband compression of HDTV, IEEE Transactions on Image Processing, IP-1:436-440, July 1992.November 7. 